\title{
Aspectos psiquiátricos do tratamento da
} obesidade

\section{Psychiatric Aspects in the Treatment of Obesity}

Fátima VAsques ${ }^{1}$

Fernanda Celeste Martins ${ }^{2}$

Alexandre Pinto de Azevedo 3

\section{Resumo}

A obesidade, por sua caracterização e etiologia multifatorial, é uma condição que tem merecido atenção e estudos de diversas áreas de especialidades, particularmente a psiquiatria e a psicologia. Os problemas emocionais são geralmente percebidos como conseqüências da obesidade, embora conflitos e problemas psicológicos possam preceder o desenvolvimento dessa condição. A depressão e a ansiedade são sintomas comuns, e depressão maior pode ser mais freqüente nos gravemente obesos. No tratamento psicoterápico, a terapia cognitiva vem mostrando eficácia por objetivar a organização das contingências para mudanças de peso e comportamentos, em princípio, relacionados ao autocontrole de comportamentos alimentares e contexto situacional mais amplo. Embora o tratamento farmacológico da obesidade não deva ser a primeira opção terapêutica, seu uso está plenamente aprovado. Atenção deve ser dada para as drogas realmente aprovadas para uso em longo prazo e as de ação central, podendo atuarem como desencadeadoras de quadros psiquiátricos.

Palavras-chave: obesidade, terapia cognitiva, agentes antiobesidade. 


\begin{abstract}
Obesity is a condition that has deserved studies of different areas, particularly psychiatry and psychology, because of its multifactorial etiology. The emotional problems generally are perceived as a consequence of obesity, but psychological conflicts and problems can precede the development of obesity. Depression and anxiety are common symptoms and depression syndrome can be more frequent in the severe obesity. The psycotherapeutic treatment, especially the cognitive therapy, shows good action for organization, weight changes and beyond the self-control of food behaviors. Although the pharmacological treatment of obesity should not be the first therapeutic option, its use is fully approved. Attention should be drawn to drugs that were really approved for use in obesity and those with central action.
\end{abstract}

Keywords: Obesity, cognitive therapy, antiobesity agents.

\section{Introdução}

A obesidade, por sua caracterização e etiologia multifatorial, é uma condição que tem merecido atenção e estudos de diversas áreas de especialidades, particularmente a psiquiatria e a psicologia. Contudo, no DSMIV-R não se encontram critérios para identificação e avaliação da obesidade como transtorno psiquiátrico, mesmo na categoria de transtornos alimentares, apesar de seus portadores apresentarem perturbações comportamentais e conflitos psíquicos relacionados à alimentação. Embora, de fato, a obesidade não deva ser classificada como transtorno alimentar, alguns autores a incluem didaticamente nesta categoria pelos aspectos de funcionamento semelhante aos demais transtornos, pelo fato de caracterizar-se por perturbações no comportamento alimentar, apresentando síndrome psicológica associada que pode merecer intervenção médica e/ou psiquiátrica (Flaherty, 1995).

\section{Compreensão da obesidade}

Há diversas formas de conceituar e classificar a obesidade. A definição primária de obesidade pode ser a de "acúmulo excessivo de tecido adiposo no organismo" (Nunes, 1998). A grande dificuldade deste conceito básico é como medir esse tecido adiposo e como estabelecer o limiar a partir do qual um determinado indivíduo será rotulado como obeso. Criou-se, então, o Índice de Massa Corporal (IMC), método mais utilizado atualmente e que define que a obesidade seria classi- ficada a partir de um IMC superior a $30 \mathrm{~kg} / \mathrm{m}^{2}$. Outra definição que se refere igualmente ao excesso de peso é a de que "uma pessoa obesa é definida, convencionalmente, como aquela que pesa $20 \%$ a mais do que o peso-padrão especificado com relação ao sexo, altura e estrutura corporal.” (Flarherty, 1995).

A cultura ocidental enfatiza mais a boa forma e a imagem corporal, o que facilita a identificação de incômodos com o excesso de peso, independente dos graus de obesidade. Nossos padrões culturais fazem com que até indivíduos com peso dentro dos parâmetros de normalidade possam sentir-se com peso acima do desejado. É possível observar a importância da participação de vários fatores etiológicos genéticos e orgânicos, da falta de atividades físicas, de fatores educacionais e psicológicos. Estes últimos, ocupando dois lugares específicos que comparticipam, lugar de causas e lugar de complicações da obesidade (Flarherty, 1995).

No aspecto clínico, relacionado a padrões de alimentação dos obesos, integram conteúdos como desconhecimento de mecanismos exatos controladores da saciedade e apetite e ainda, em alguns, com visão negativa do corpo, preocupados com a forma como responsável pelo acesso, aceitação, sucesso social e felicidade.

Os problemas emocionais são geralmente percebidos como conseqüências da obesidade, embora conflitos e problemas psicológicos de autoconceito possam preceder o desenvolvimento da obesidade. Adepressão e a ansiedade são os sintomas comuns; depressão maior pode ser freqüente nos gravemente obesos. Pacientes obesos emocionalmente instáveis podem experienciar 
aumento na ansiedade e depressão quando fazem dietas (Flarherty, 1995). Portanto, o obeso apresenta aspectos emocionais e psicológicos identificados como causadores ou conseqüências ou retroalimentadores da sua condição de obeso, concomitante a uma condição clínica e educacional alterada.

\section{Aspectos psicológicos do tratamento da obesidade}

No tratamento psicoterápico, a terapia cognitiva vem mostrando eficácia por trabalhar a partir da estrutura operante do paciente com objetivos de organizar as contingências para mudanças de peso e comportamentos, em princípio, relacionados ao autocontrole de comportamentos alimentares, e contexto situacional amplo, aprofundando para todo o desconforto (Abreu, 2003). A avaliação e correção dos pensamentos inadequados, que contribuem tanto para a etiologia quanto para a manutenção da obesidade, são procedimentos disparadores e freqüentes no processo psicoterapêutico para a modificação comportamental. A reestruturação cognitiva, imagens orientadas, o treinamento da auto-instrução, a determinação de objetivos, o estímulo ao auto-reforço e resolução de problemas são alguns procedimentos inter-relacionados, de base cognitiva, incorporados a outros programas comportamentais (Abreu, 2003).

A orientação cognitivo-comportamental segue o modelo que identifica a crença central e a crença intermediária (regra, atitude, suposição) que leva a um pensamento e influencia uma situação, e viceversa, desencadeando igualmente reações emocionais, comportamentais e fisiológicas (Hawton, 1997). Com base nesta orientação, os sistemas de crenças de indivíduos obesos determinam sentimentos e comportamentos desencadeados por pensamentos disfuncionais acerca do peso, da alimentação e do valor pessoal; por exemplo, a crença de que ser magro está associada a autocontrole, competência e superioridade interfere diretamente na constituição da auto-estima da pessoa, ou mesmo, a crença de que ser magro é fundamental para a solução de problemas da vida e que, portanto, pessoas obesas seriam infelizes e malsucedidas, são significações que também são encontradas neste grupo (Abreu, 2003).

Estes conjuntos de crenças provocam, no obeso, tendências disfuncionais de raciocínio levando-o a desenvolver pensamentos dicotômicos - pensamentos em termos absolutos e extremos do tipo "se não estou completamente com o controle, significa que perdi todo o controle, que está tudo perdido; então, posso me fartar." Considerando-se o sistema de crenças, identificamos os aspectos psicológicos da obesidade, aspectos envolvidos no controle da alimentação, ou seja: as correlações, interdependências e interações que existem entre o ambiente, pensamentos, sentimentos e comportamentos (Hawton, 1997).

Portanto, o desafio da psicoterapia cognitiva é compreender como diversos fatores interagem entre si em cada caso ou situação e, associada e integrada a outras terapias, favorecer a melhora no manejo do sintoma para que o paciente possa dispor de um repertório qualitativamente mais amplo para responder às demandas da vida.

Na obesidade, nem sempre o tratamento farmacológico é a primeira opção terapêutica; este deve, antes, compor o tratamento que deve ser pautado numa abordagem multidisciplinar. Dietoterapia associada à psicoterapia, por serem modalidades nãoinvasivas, devem ser sempre priorizadas. No entanto, quando transtornos psiquiátricos como os transtornos fóbico-ansiosos, depressão atípica, síndrome do comer noturno e/ou transtorno da compulsão alimentar periódica (TCAP) estão presentes contribuindo para o ganho de peso, devemos considerar a farmacoterapia (Aronne, 2003).

\section{Drogas aprovadas para uso em longo prazo}

A sibutramina é uma amina terciária que age inibindo a recaptação de serotonina e noradrenalina e, em menor grau, de dopamina. É usualmente utilizada em doses de 10 a $15 \mathrm{mg} / \mathrm{dia}$. Atua diminuindo a ingestão alimentar e deve elevar a termogênese em alguns indivíduos (Aronne, 2003). Estudos mostram que $66 \%$ daqueles que completam um ano de tratamento perdem mais de $5 \%$ de seu peso comparados a apenas $29 \%$ do grupo placebo. Cerca de $39 \%$ dos pacientes perderam mais que $10 \%$ do peso comparados com 6\% do controle (Bray, 1999). A sibutramina foi inicialmente desenvolvida para uso antidepressivo (Aronne, 2003) e, embora seja descrita alguma ação em quadros de depressão maior, não deve ser utilizada para esta finalidade. Além disso, atenção especial deve ser dada ao uso em indivíduos cuja ação de maior disponibilidade de neurotransmissores deva ser evitada, como os portadores de transtorno afetivo bipolar. Efeitos colaterais como irritabilidade e inquietação podem ocorrer.

O orlistat é um agente farmacológico que inibe a lipase pancreática, diminuindo a absorção de gordura no trato gastrintestinal e promovendo a perda de peso. $\mathrm{O}$ uso dessa medicação implica uma dieta orientada, e sua associação com exercícios físicos potencializa os resultados. Contudo, não apresenta efeito supressor de episódios de compulsão alimentar, já que não há ação central (Hvizdos, 1999). Além disso, por sua ação exclusivamente gastrintestinal e por não promover alterações na absorção de drogas usualmente utilizadas em psiquiatria como os antidepressivos, pode ser a droga de escolha em indivíduos obesos em tratamento psiquiátrico. 
Drogas como a fluoxetina e sertralina não foram aprovadas para tratamento da obesidade. Reganho de peso costuma ocorrer após seis meses do início do tratamento. Essas drogas, contudo, podem ser benéficas em casos de comorbidades, como o transtorno da compulsão alimentar periódica (Aronne, 2003).

\section{Drogas utilizadas para tratamento em curto prazo}

Medicamentos anorexígenos de ação central, geralmente hipotalâmica nos sistemas catecolaminérgicos (noradrenalina e/ou dopamina), são conhecidos por serem derivados quimicamente das anfetaminas. São drogas que devem ser utilizadas por curto prazo tanto por seus potenciais efeitos colaterais como por seu elevado potencial para abuso e dependência, além da possibilidade de desencadear sintomas e síndromes psiquiátricas, como surtos psicóticos, síndromes depressivas ou de mania.

\section{Drogas com potencial futuro para uso na obesidade}

O topiramato é uma droga anticonvulsivante e tem sido estudado como medicação promissora no tratamento da obesidade. Inicialmente utilizado como estabilizador do humor e no controle da impulsividade, observou-se sua propensão à promoção da perda de peso e no tratamento do transtorno da compulsão alimentar periódica. Estudos demonstraram, em populações obesas, redução importante dos episódios de compulsão alimentar e posterior perda de peso (Shapira, 2000). História pessoal de transtorno depressivo diagnosticado ou sintomas depressivos importantes contra-indicam seu uso.

\section{Conclusão}

Quando o indivíduo obeso apresenta comorbidade do espectro psiquiátrico, a associação medicamentosa à dietoterapia e psicoterapia torna-se imprescindível, ficando bem indicado o uso de antidepressivo associado ou não com estabilizador de humor. Os agentes antiobesidade são mais indicados para obesidade sem comorbidade psiquiátrica e como coadjuvantes na presença de comorbidades. As drogas anorexígenas, apesar de eficazes, devem ser usadas com cautela e por curto período de tempo. Apesar de o tratamento do ponto de vista psiquiátrico da obesidade estar em evidência, é necessário maior investimento no desenvolvimento do acompanhamento em longo prazo.

\section{Referências bibliográficas}

Abreu, C.N.; Roso, M. e cols.- Psicoterapias Cognitiva e Construtivista, novas fronteiras da prática clínica. Artmed, Porto Alegre, 2003.

ARONNE, L.J.- Current pharmacological treatments for obesity. In: Fairbuirn \& Brownell (Eds).- Eating disorders and obesity. 2.ed. Guilford Press, New York, 2003, p. 551-6.

Beck, A.; Freeman, A. e cols.- Terapia cognitiva dos transtornos de personalidade. Artes Médicas, Porto Alegre, 1993.

Bray G.A.; Greenway, F.L.- Current and potential drugs for treatment of obesity. Endocrine Reviews 20: 805-75, 1999.

CID-10 - Classificação de transtornos mentais e de comportamento. Artes Médicas, Porto Alegre, 1993.

DSM-IV-TR- Manual diagnóstico e estatístico de transtornos mentais. Artmed, Porto Alegre, 2002.
Flaherty, D. \& Janicak, P.G. Psiquiatria, diagnóstico e tratamento. Artes Médicas, Porto Alegre, 1995.

Freemam, A.; Dattilio, F.M.- Compreendendo a terapia cognitiva. Editorial Psy, São Paulo, 1998.

Hawton, K.; Salkovskis, P.M.; KIRK, J.; ClaRK, D.M. - Terapia cognitivo comportamental para problemas psiquiátricos, um guia prático. Martins Fontes, São Paulo,1997.

Hvizdos K.M.; MARKHAM, A.- Orlistat: a review of its use in the management of obesity. Drugs 58: 1093-124,1999.

Nunes, M.A.A.; Apolinário, J.C.; Abuchaim, A.L.G.; Coutinho W. e cols. - Transtornos alimentares e obesidade. Artmed, Porto Alegre, 1998.

ShapiRa N.A.; Golsmith, T.D.; McelroY, S.L.- Treatment of binge eating disorder with topiramate: a clinical case series. J Clin Psychiatry 61: 368-72, 2000. 